\title{
A New Adaptive Imaging Technique Using Generalized Coherence Factor
}

\author{
Meng-Lin Li and Pai-Chi Li \\ Department of Electrical Engineering, National Taiwan University, \\ Taipei, Taiwan, R. O. C.
}

\begin{abstract}
Beamforming artifacts due to sound velocity inhomogeneities degrade both spatial and contrast resolution. In this paper, a new adaptive imaging technique is proposed to minimize such beamformation artifacts using the generalized coherence factor (GCF) weighting. The simulation and experimental results show that the proposed technique can significantly improve the degraded image quality due to phase aberration for both point targets and diffuse scatterers. The GCF technique is also compared with the correlation-based technique and the parallel receive beam technique (a.k.a PARCA). Both performance and computational complexity are also discussed.
\end{abstract}

\section{INTRODUCTION}

For diagnostic ultrasound systems, time delay errors due to sound velocity inhomogeneities are likely to produce beamformation artifacts that degrade both spatial and contrast resolution. Such effects are also known as phase aberrations, and many approaches have been proposed for phase aberration correction. The correlation-based technique has been the most widely explored technique for the correction of phase aberrations [1]. Correlation functions of signals either from adjacent channels or from individual channels and the beamsum are typically used to find out the time delay errors. Then, the estimated time delay errors are applied to correct the transmit and receive focal delays. However, such time delay estimation is computationally intensive and the performance is limited.

The parallel receive beam technique (PARCA) is an alternative phase-aberration correction approach [2]. PARCA does not directly estimate propagation parameters, but instead attempts to minimize focusing errors irrespective of the source of the artifacts. However, since PARCA needs to solve the total least-squares problem, it is very computationally intensive and hence is difficult to implement in real time. Therefore, a modified algorithm using Fourier-transform-based parallel beam reconstruction (a.k.a. PARCA2) has been proposed for performing aberration correction much more efficiently [3]. In addition, based on the relationship between parallel receive beams and the Fourier spectrum over the aperture, the sidelobe filter and the null filter were proposed to improve the beam quality with a point target [4].

This paper proposes a more efficient approach based on the generalized coherence factor (GCF). The GCF is an index of the beamforming quality and is used as an adaptive weighting factor to improve degraded image quality in this paper. When the GCF is high the focusing quality is good, and thus the weighting is high so that image intensity is maintained. On the other hand, the focusing quality is low when the GCF is low, and hence the weighting needs to be low to reduce the artifacts. In this paper, the effectiveness of adaptive GCF weighting is demonstrated. In addition, it is shown that the performance of the proposed GCF technique rivals that of existing approaches.

\section{GENERALIZED COHERENCE FACTOR}

The GCF used as a weighting factor is derived from the spectrum of the received channel data along the array direction after proper receive delays have been applied. Specifically, the GCF is defined as the ratio of the spatial spectral energy within a prespecified low-frequency region to the total energy. The GCF over the aperture at a given range can be expressed as

$$
\mathrm{GCF}=\frac{\sum \in \text { prespified low-frequency region }}{\sum_{k=0}^{N-1}|p(k)|^{2}},
$$

where $p(k)$ is the discrete Fourier transform of the received array data along the array direction, $N$ is the length of $p(k)$. The discrete Fourier transform 
can be efficiently computed using the fast Fourier transform (FFT), and $N$ is equal to the number of channels in this case. The low-frequency region is specified by a cutoff frequency $M_{0}$. Note that an $M_{0}$ of zero means that the low-frequency region used to estimate GCF is restricted to dc. The procedure is illustrated in Fig. 1.

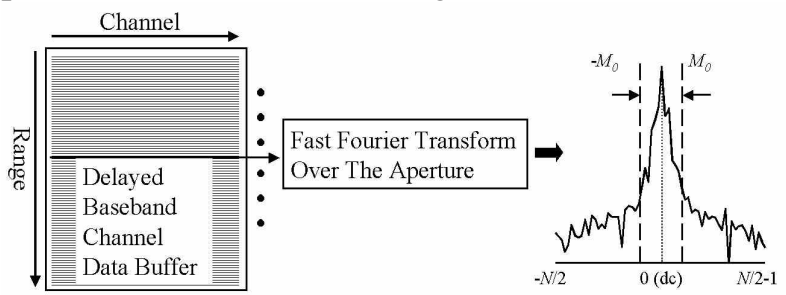

Fig. 1 Schematic diagram showing how the GCF is calculated.

The dc component of the spectrum is also the coherent sum. The total spectral energy, on the other hand, is $N$ times the incoherent sum. Thus, if the prespecified low-frequency region is restricted to dc only, the GCF reduces to the coherence factor defined in the literature [5]. Since the low-frequency component generally corresponds to the coherent portion of the received data, and the high-frequency component corresponds to the incoherent portion, the GCF can be viewed as an index of the focusing quality.

A high GCF corresponds to good focusing quality, and thus the image intensity should be maintained. A low GCF, on the other hand, should be used to reduce the contribution of the data to the image intensity because significant beamforming errors are present. Hence, the GCF is used as a weighting factor to improve the degraded beam quality due to phase aberrations. The weighting is done by multiplying the amplitude of the beam sum data by the corresponding GCF for each image point. The corrected signal $x_{\text {corrected }}$ of $x$ at a particular range can be expressed as

$$
x_{\text {corrected }}=\mathrm{GCF} \cdot x \text {. }
$$

Note that with a speckle-generating target, the returning echo from scatterers within a sample volume inherently contains a certain degree of incoherence. Thus, the low-frequency range cannot be restricted to only dc for such a target.

\section{SIMULATION RESULTS}

In this section, simulated ultrasound data are used to explore the efficacy of the GCF-weighting technique. The simulated phased array had 64 elements with a center frequency of $3.5 \mathrm{MHz}$, and a half-wavelength pitch. The transmit focal point was $30 \mathrm{~mm}$ from the transducer, and dynamic focusing was applied on receive. Only a near-field phase screen was simulated for all aberrated cases.

The normalized aberration profile is shown in Fig. 2. The correlation length of the profile is 5 $\mathrm{mm}$. For all aberrated cases, the aberration profile was applied on both transmit and receive. Two types of image objects - a point target and an anechoic object - were simulated.

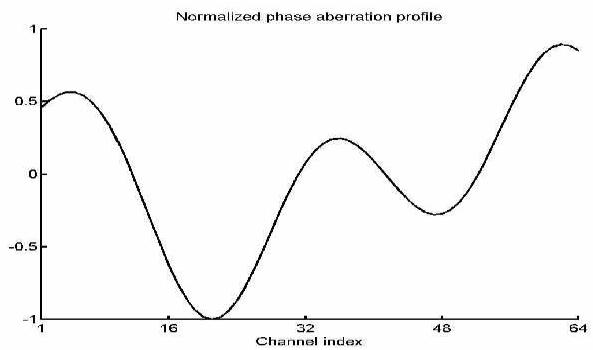

Fig. 2 The normalized phase-aberration profile.

\section{A. Point Target}

A point target is located at $30 \mathrm{~mm}$, which is also the transmit focal point. Fig. 3 shows the projected beam patterns for the point target under different aberration conditions and different values of $M_{0}$. Figs. 3(a) and (b) show the aberrated cases with $\pi / 4$, and $\pi / 2$ maximum phase errors, respectively, at the imaging frequency of $3.5 \mathrm{MHz}$. It is obvious that the beam quality is significantly improved after adaptive weighting for each aberration case and $M_{0}$ value: the near sidelobes are suppressed by $8 \mathrm{~dB}$ and the far sidelobes are all significantly suppressed by more than $20 \mathrm{~dB}$. Note that the efficacy of the proposed technique decreases as the maximum phase error and $M_{n}$
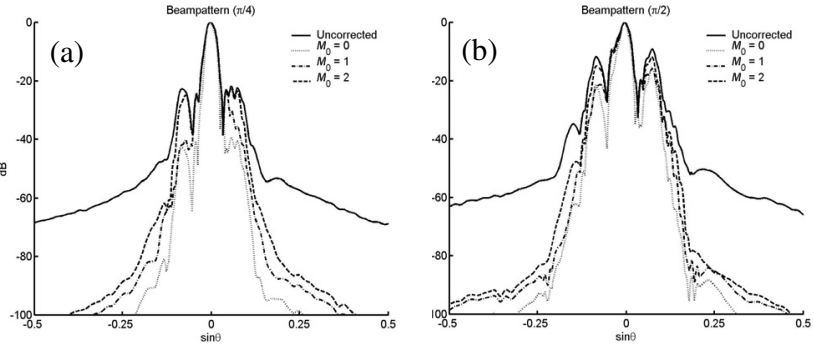

Fig. 3 Projected beam patterns for a point target. (a) and (b) are $\pi / 4$, and $\pi / 2$ maximum phase errors, respectively, at the center frequency of $3.5 \mathrm{MHz}$.

\section{B. Anechoic Object}

To demonstrate the efficacy of the proposed GCF technique for targets with diffuse scatterers and contrast resolution, an anechoic cyst in a speckle-generating background is simulated. The 
center of the cyst is located at the transmit focal depth of $30 \mathrm{~mm}$. The positions of the scatterers are uniformly distributed, and the scattering amplitudes are Gaussian distributed.

Figs. 4 show the uncorrected and GCF-corrected images of a phantom with an anechoic cyst. Note that the images are sector-scan images prior to scan conversion, and display over a $50 \mathrm{~dB}$ dynamic range. The vertical axis is the range and the horizontal axis represents the azimuth. The top row shows the case with $\pi / 4$ maximum phase error and the bottom row is the case with $\pi / 2$ maximum phase error. Figs. $4(b)$ and (e) shows the results weighted by GCF with $M_{0}=0$. Figs. 4(c) and (f) are the results weighted by GCF with $M_{0}=1$. It is obvious that the detection of the cyst is significantly improved, but the adaptive weighting technique is less effective for the case with a $\pi / 2$ maximum phase error. However, the corrected images with $M_{0}=0$ exhibit artificial black holes, and large image intensity variations are introduced. Such results suggest that it is inappropriate to set $M_{0}$ to zero (i.e., the coherence factor) for diffuse scatterers. A larger $M_{0}$ is generally more preferable for this type of object. That is, the low-frequency region used to estimate GCF for diffuse scatterers should be wide enough to include the inherent incoherence from a speckle-generating target.

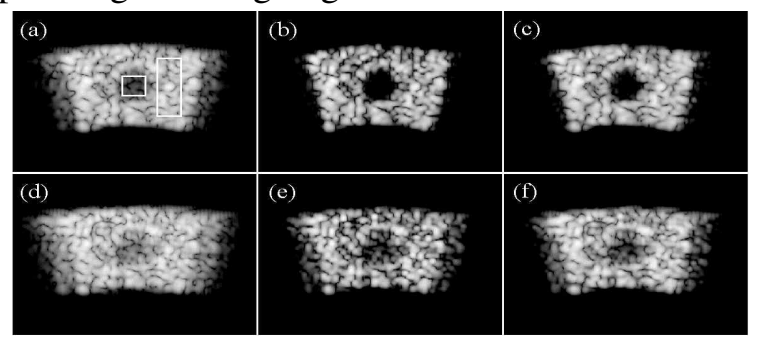

Fig. 4 Original and GCF-corrected images of a phantom with an anechoic cyst. Top row shows the case with $\pi / 4$ maximum phase error. Bottom row are the case with $\pi / 2$ maximum phase error. (a) and (d): uncorrected. (b) and (e): corrected with $M_{0}=0$. (c) and (f): corrected with $M_{0}=1$.

Two indices related to contrast resolution are employed to evaluate the improvement in image quality: (i) the contrast ratio (CR) is defined as the ratio of the mean value in the background to the mean value in a cyst region, and (ii) the contrast-to-noise ratio (CNR) is defined as the CR divided by the standard deviation of image intensity in the background region [2] (note that the background and cyst regions are indicated by the right- and left-hand white boxes shown in Fig. 4(a), respectively). The GCF-corrected images with $M_{0}=1$ exhibit contrast improvements of 15.74 $\mathrm{dB}$, and $8.64 \mathrm{~dB}$ for maximum phase errors of $\pi / 4$, and $\pi / 2$, respectively. A higher CNR is also obtained after adaptive GCF weighting with $M_{0}=1$, and the improvement ranges from $39 \%(\pi / 4)$ to $18 \%(\pi / 2)$.

\section{EXPERIMENTAL RESULTS}

Emulated images using real ultrasound data are presented in this section. Three complete RF data sets with three different levels of distortion were used to test the efficacy of the proposed adaptive weighting method. The first data set was $\mathrm{RF}$ data acquired from a tissue-mimicking phantom without distortion. The other two data sets were acquired from the same phantom except that $1 \times$ and $2 \times$ distortion were introduced, corresponding to maximum phase errors of $\pi$ and $2 \pi$, respectively, at the imaging frequency of 3.33 MHz. They were acquired using a 64-element, $3.33-\mathrm{MHz}$ phased-array transducer with a 17.76-MHz sampling frequency. Dynamic receive focusing with a f/number of 2 was applied, and the transmit focus was at $60 \mathrm{~mm}$. All images are displayed over a $50 \mathrm{~dB}$ dynamic range and are shown in a prescan conversion format.

Fig. 5(a) is the image without distortion, and Figs. 5(b) and (c) show images of the phantom with $1 \times$ and $2 \times$ distortion, respectively. Figs. 5(d)-(f) show the corrected images of Figs. 5(a)-(c), respectively, using GCF weighting with $M_{0}$ set to 2 . Note that all the images are normalized by the mean value of the homogeneous region located at the image center. These corrected images show that the image quality is noticeably improved. CR and CNR are calculated using the background and cyst region indicated by the black and white boxes shown in Fig. 5(a). Using GCF, the corrected images have contrast enhancements of $15.23 \mathrm{~dB}$ (no distortion), $11.11 \mathrm{~dB}(1 \times)$, and $7.05 \mathrm{~dB}(2 \times)$. Higher CNR values are also obtained after adaptive weighting, and the improvement ranges from $41 \%$ (no distortion) to $16 \%(2 \times)$. The corrected images with less distortion have both higher $\mathrm{CR}$ and higher $\mathrm{CNR}$ values.

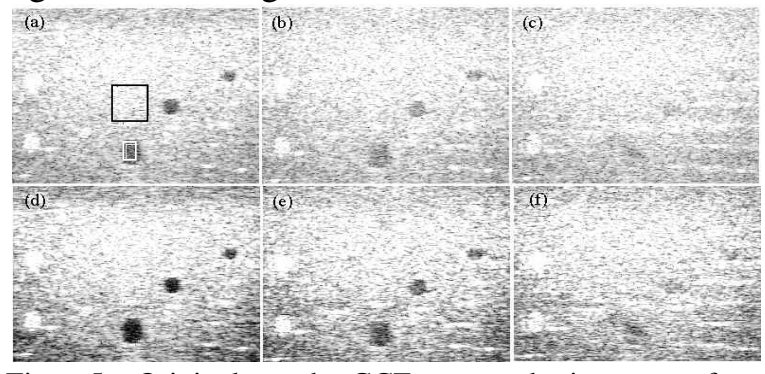

Fig. 5 Original and GCF-corrected images of a tissue-mimicking phantom over a $50 \mathrm{~dB}$ dynamic range. Top row: uncorrected; bottom row: corrected. (a) and (d): no distortion. (b) and (e): $1 \times$ distortion. (c) and (f): $2 \times$ distortion. 


\section{DISCUSSION}

The simulation results show that the cutoff frequency $M_{0}$ affects the performance of the GCF technique. The GCF technique with an $M_{0}$ of zero is only suitable for point targets; for diffuse scatterers, $M_{0}$ should be sufficiently large in order not to introduce image artifacts. However, the performance of the GCF technique is degraded when $M_{0}$ is too large.

The performance of the proposed GCF technique with a displaced phase screen was also evaluated, and compared with the correlation-based technique [1] and PARCA2 [3]. The displaced phase-screen profile is the same as the normalized phase-aberration profile shown in Fig. 2. The maximum phase error is $\pi / 2$ at the imaging frequency of $3.5 \mathrm{MHz}$. A point source is located at a depth of $80 \mathrm{~mm}$, with the transducer being focused at the same depth; the simulation results are shown in Fig. 6. Fig. 6 shows the receive beam patterns with the phase screen at a depth of $40 \mathrm{~mm}$. The lateral axis is azimuth. Since a point target is imaged, $M_{0}$ was chosen as 0 for GCF calculations.

Fig. 6(a) compares the GCF and correlation-based techniques. It is shown that the GCF technique performs well for a displaced phase screen, even outperforming the correlation-based technique for the displaced phase screen situation. Fig. 6(b) compare the GCF technique and PARCA2 (three iterations were used for PARCA2). It is shown that the GCF technique also outperforms PARCA2 for the displaced phase-screen situation. Compared to PARCA2, the GCF technique has lower computational complexity and memory requirements, and no iterations are needed.

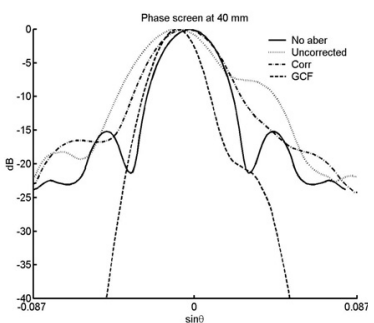

(a)

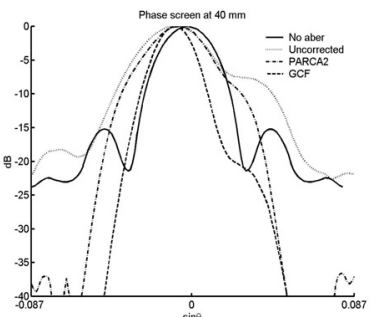

(b)
Fig. 6 Projected receive beam patterns for a point target with a displaced phase screen. Solid: no aberration; dotted: uncorrected; dashed: corrected by GCF weighting; dashed-dotted: corrected by the correlation-based technique in (a), and corrected by PARCA2 in (b).

\section{CONCLUSIONS}

In this paper, the new imaging technique using GCF adaptive weighting has been tested for the correction of phase aberration. The simulation and experimental results demonstrate that the proposed GCF technique could potentially correct for both near field and distributed aberrations in the presence of both point targets and diffuse scatterers. In addition, it is shown that the performance of the proposed GCF technique is subject to the cutoff frequency $M_{0}$.

Compared to the parallel receive beam technique, the proposed GCF technique has better performance. In addition, the computational complexity and system memory requirement for the GCF technique is lower, and no iteration is needed. The performance of the proposed technique is also compared with that of the correlation-based technique for a displaced phase screen. It is also shown that the proposed GCF technique performs better than the correlation-based technique in the presence of a mid-range phase screen.

\section{REFERENCES}

[1] S. W. Flax and M. O'Donnell, "Phase-aberration correction using signals from point reflectors and diffuse scatterers: basic principles," IEEE Trans. Ultrason., Ferroelect., Freq. Contr., vol. 35, no. 6, pp. 758-767, 1988.

[2] S. Krishnan, P.-C. Li, and M. O'Donnell, "Adaptive compensation of phase and magnitude aberrations," IEEE Trans. Ultrason., Ferroelect., Freq. Contr., vol. 43, no. 1, pp. 44-55, 1996.

[3] S. Krishnan, K. W. Rigby, and M. O'Donnell, "Efficient parallel adaptive aberration correction," IEEE Trans. Ultrason., Ferroelect., Freq. Contr., vol. 43, no. 1, pp. 691-703, 1998.

[4] M. K. Jeong, "A Fourier transform-based sidelobe reduction method in ultrasound imaging," IEEE Trans. Ultrason., Ferroelect., Freq. Contr., vol. 47, no. 3, pp. 759-763, 2000.

[5] K. W. Hollman, K. W. Rigby, and M. O'Donnell, "Coherence factor of speckle from a multi-row probe," in Proc. IEEE Ultrason. Symp., 1999, pp. 1257-1260. 